\title{
Coronavirus Pandemic: Could Nature's Bounty Combat the Disease and Other Similar Infections
}

\author{
Basit L Jan MS and Khalid M Alkharfy PharmD, PhD* \\ Department Clinical Pharmacy, College of Pharmacy, King Saud University, Riyadh 11451, Saudi Arabia \\ ${ }^{\star}$ Corresponding Author: Khalid M Alkharfy, Department Clinical Pharmacy, College of Pharmacy, King Saud University, Riyadh 11451, Saudi Arabia; \\ E-mail: alkharfy@ksu.edu.sa
}

Received: March 06, 2020; Accepted: March 11, 2020; Published: March 13, 2020

As the coronavirus epidemic in China continues to spread to other parts of the world, so far infecting at least 98,000 and killing over 3,000 people (6 March 2020; source: www.cbsnews.com), scientists and clinicians all over the world are racing against time to find a treatment. People infected with the novel coronavirus, named COVID-19, have not received a treatment specific to this virus since none yet exists. In-fact, so far no treatment has been approved for any of the coronaviruses known to infect humans. Rather, infected subjects are treated symptomatically with respiratory support, fever reduction, and rehydration; as well as, managing associated complications [1].

Coronavirus infection symptoms range from fever, cough and struggling to breath, to the much more serious cases of acute respiratory distress syndrome, pneumonia, kidney failure and death [2]. People with an already compromised immunity and elderly are considerably at more risk of getting infected with coronavirus and developing severe disease according to the USA Center for Disease Control and Prevention (CDC). A human-to-human transmission occurs through personal contact with an infected person, sneezing, coughing, touching contaminated surfaces and secretions of the mouth, nose or eyes. In very rare cases, transmission via fecal spread may take place. CDC recommends basic hygiene techniques for respiratory viruses such as washing hands regularly, sanitizing surfaces constantly, coughing into one's arm or a tissue, keeping yourself hydrated and avoiding contact with one's face or anyone who is sick. Moreover, wearing proper masks, gloves and gowns when dealing with a suspected or infected person.

Viral infections are usually difficult to treat. This is because viruses are very diverse, with unique characteristics, allowing for constant genetic mutations that can impart resistance to available antiviral drugs. Targeting the viruses while not harming human cells can be challenging, since viruses use human cellular mechanisms to form proteins that help it to reproduce $[3,4]$. Although some pharmaceutical companies are already working on various possible anti-viral therapies to treat the new coronavirus, it is going to take some time for such drugs to be tested and approved.

Herbal remedies have been documented to cure infectious diseases for almost 2,000 years (5), with more than 10,000 herbal medicines and 100,000 recipes recorded in ancient literatures [6], giving us a very rich source to screen for pharmacological activities. Herbal products have especially been used to treat and inhibit viral respiratory infections (VRI) [5]. Rhinovirus, coronavirus, meta- pneumovirus, para-influenza, adenovirus, enterovirus, respiratory syncytial virus (RSV) and influenza viruses are the major causes of VRI [5]. Herbs tend to exhibit less side-effects and mild cured process as compared to other anti- infection drugs [7]. The benefits of herbs having integral immune-stimulating and inflammation-modulating influence means that they can help inhibit immune over-reaction (cytokine storm) to VRI while still helping the immune system manage better with the infections [8].

Generally, no single herb's constituent is a solution to VRI, but instead a range of components with diverse actions are needed. Perhaps most important among those are herbs working against the invading viruses directly and improving human immune-system against infections. As such, it seems the circulation and expression of cytokines and their receptors are monitored by various immune connected cells under the stimuli of herbs [9]. For centuries, roots of Pelargonium sidoides have been used for treating diverse illnesses including infections of the airways in the southern Africa region [10-12]. In Germany and some Middle East countries, Pelargonium sidoides extract products such as $\mathrm{EPs}^{\circledR} 7630$ and Plerus ${ }^{\oplus}$, have been approved and marketed as a therapy for the treatment of acute bronchitis. It prevents the replication of multiple respiratory viruses including respiratory syncytial virus (RSV), seasonal influenza-A virus strains, parainfluenza virus, human coronavirus and coxsackie virus. The underlying beneficial effects of the Pelargonium sidoides extract in bronchitis patients are postulated to include immunemodulatory and cyto- protective effects, prevention of interaction between the infectious agent and host cells and increase of ciliary beat frequency on respiratory cells $[10,12]$.

Other herbal products have been used as adjuvants for medicinal enhancement. Polygala tenuifolia root extract has been reported to exhibit a robust mucosal adjuvant activity [13]. The active adjuvant substances were isolated and identified as onjisaponins A, E, F and G. Onjisaponins have been shown to enhance the levels of serum antibody and nasal anti-influenza virus IgA and IgG when co-immunized with vaccines for influenza virus compared to inoculation of vaccines alone. In addition, intra-nasal vaccination with onjisaponin $F$ has been shown to reduce the activity of mouse-adapted influenza virus A/PR/8/34 (H1N1) in broncho-alveolar lavages of mice [13]. Another adjuvant remedy, 9S, 12S, 13S-trihydroxy-10E-octadecenoic acid (pinellic 
acid), which is isolated from the tuber of Pinellia ternate Breitenbach, has been shown as an effective oral adjuvant for the nasal influenza vaccine [14]. As a traditional herb against influenza, the compounds of Astragalus membranaceus have been well researched and the saponins extracted from Astragalus membranaceus significantly enhanced the proliferation of ovalbumin induced splenocyte and antibody titers of ovalbumin specific IgG, IgG1 and IgG2b in serum, demonstrating the effective adjuvant function of saponins [15].

In summary, more translational and clinical studies are required to explore the promising effects of herbal products in the treatment and prevention of viral infections particularly those affecting the respiratory system.

\section{References}

1. McKimm-Breschkin JL, Jiang S, Hui DS, Beigel JH, Govorkova EA, Lee N (2018) Prevention and treatment of respiratory viral infections: Presentations on antivirals, traditional therapies and host-directed interventions at the 5th ISIRV Antiviral Group conference. Antiviral Res 149: 118-142. [crossref]

2. Chafekar A, Burtram C Fielding (2018) MERS-CoV: Understanding the Latest Human Coronavirus Threat. Viruses 10: 93.

3. Fredric S Cohen (2016) How Viruses Invade Cells. Biophys $J$ 110: 1028-1032. [crossref]

4. White JM, Delos SE, Schornberg K (2008) Structures and mechanisms of viral membrane fusion proteins: multiple variations on a common theme. Crit Rev Biochem Mol Biol 43: 189-219. [crossref]

5. Eric Yarnell (2018) Herbs for Viral Respiratory Infections. Alternative and complementary therapies. 24: 1.

6. T Li, T Peng (2013) Traditional Chinese herbal medicine as a source of molecules with antiviral activity. Antiviral Research 97: 1-9.

7. Shen B (2015) A new golden age of natural products drug discovery. Cell 163: 1297-1300. [crossref]

8. Donkor PO, Chen Y, Ding LQ, Qiu F (2016) Locally and traditionally used Ligusticum species-A review of their phytochemistry, pharmacology and pharmacokinetics. J Ethnopharmacol 194: 530-548.

9. Wang H, Actor JK, Indrigo J, Olsen M, Dasgupta A (2003) Asian and Siberian ginseng as a potential modulator of immune function: an in vitro cytokine study using mouse macrophages. Clin Chim Acta 327: 123-128.

10. Brendler T, van Wyk BE (2008) A historical, scientific and commercial perspective on the medicinal use of Pelargonium sidoides (Geraniaceae). $J$ Ethnopharmacol 119: 420-433.

11. Kolodziej H (2008) Aqueous ethanolic extract of the roots of Pelargonium sidoides - new scientific evidence for an old anti-infective phytopharmaceutical. Planta Med 74: 661-666.

12. Wang Xiaoguang and Liu Zejing (2014) Prevention and treatment of viral respiratory infections by traditional Chinese herbs. Chinese Medical Journal 127:1344-1350.

13. Nagai T, Kiyohara H, Munakata K, Shirahata T, Sunazuka T, et al. (2002) Pinellic acid from the tuber of Pinellia ternate Breitenbach as an effective oral adjuvant for nasal influenza vaccine. Int Immunopharmacol 2: 1183-1193.

14. Yang ZG, Sun HX, Fang WH (2005) Haemolytic activities and adjuvant effect of Astragalus membranaceus saponins (AMS) of the immune responses to ovalbumin in mice. Vaccine 23: 5196-5203.

15. Shimizu T, Tomioka H, Sato K, Sano C, Akaki T, et al. (1999) Effects of the Chinese traditional medicine mao-bushi-saishinto on therapeutic efficacy of a new benzoxazinorifamycin, KRM-1648, against Mycobacterium avium infection in mice. Antimicrob Agents Chemother 43: 514-519.

\section{Citation:}

Basit L Jan MS, Khalid M Alkharfy PharmD, PhD (2020) Coronavirus Pandemic: Could Nature's Bounty Combat the Disease and Other Similar Infections. Microbiol Immunol Pathol, Volume 1(1): 1-2. 